\title{
Reconstruction d'écoulements incompressibles à partir de données lagrangiennes
}

\author{
Yann Brenier ${ }^{*}$ et Michel Roesch ${ }^{\dagger}$
}

Résumé

La reconstruction du champ de vitesse d'un écoulement dont on connait certaines données lagrangiennes est un problème fréquemment rencontré dans de nombreuses applications (reconstitution du vent à partir de cartes de concentration en météorologie, du courant marin à partir de trajectoires de bouées en océanographie, interpolation d'images, etc...). Le plus souvent l'écoulement est régi par des équations de mécanique des fluides plus ou moins complexes, mais en définitive issues des équations de Navier-Stokes ou d'Euler et, assez souvent, en régime incompressible. Dans ce travail, on montre comment, sur un intervalle de temps fixé, on peut opérer une reconstruction d'écoulements incompressibles non visqueux à partir de données lagrangiennes plus ou moins complètes aux instants initial et final, par simple minimisation de l'énergie cinétique, où les équations d'Euler apparaissent naturellement comme conditions d'optimalité formelles. Quelques méthodes numériques, étudiées en détail dans la thèse de Michel Roesch [13], sont brièvement présentées dans le cas de données complètes.

\section{Présentation}

On considère le cas d'un écoulement d'un fluide incompressible à l'intérieur d'un domaine compact $D$ de $\mathbb{R}^{d}$ qui peut être soit l'adhérence d'un ouvert borné assez régulier soit un domaine périodique tel que $\mathbb{T}^{d}=(\mathbb{R} / \mathbb{Z})^{d}$. L'intervalle de temps considéré est fixé. On le note $[0, T]$ et on pose $Q=[0, T] \times D$. A chaque instant $t$, le champ de vitesse $x \in D \rightarrow u(t, x) \in$ $\mathbb{R}^{d}$ est à divergence nulle (hypothèse d'incompressibilité) et, pour simplifier, supposé soit tangent au bord de $D$ dans le cas d'un domaine à bord, soit d'intégrale nulle sur $D$ dans le cas d'un domaine périodique. On considère alors deux problèmes, l'un très académique dit de "données lagrangiennes complètes", l'autre plus réaliste dit de "données lagrangiennes incomplètes". Dans le cas de données complètes, on suppose connue la position $h(a) \in D$ à l'instant final $t=T$ de toute particule fluide issue du point $a \in D$ à l'instant initial $t=0$. Dans le cas de données incomplètes, on suit l'évolution d'un (ou plusieurs) "traceur" $c(t, x)$, transporté par le fluide, et dont on suppose connues les valeurs aux instants $t=0$ et $t=T$. Dans les deux cas, on veut reconstruire, moyennant des conditions de compatibilité sur les données, un écoulement qui obéisse aux équations d'Euler des fluides parfaits incompressibles, simplement en minimisant l'énergie cinétique $\int_{Q}|u(t, x)|^{2} d t d x$ parmi tous les champs de vitesse admissibles. C'est à l'étude mathématique et numérique de ce problème variationnel qu'est consacré cet exposé.

*Université Paris 6 et ENS, DMI, 45 rue d'ULM, 75230 Paris Cedex, France.

técole des Mines de Paris, France. 


\section{Problèmes et résultats théoriques}

\subsection{Le cas de données lagrangiennes incomplètes}

\section{Définition d'un traceur}

Un traceur sera, pour nous, une fonction $c(t, x)$ à valeurs binaires 0 ou 1 (pour presque tout $(t, x) \in Q$ ) satisfaisant à la "loi de conservation" (ou "équation de continuité", suivant le jargon utilisé)

$$
\partial_{t} c+\nabla \cdot(c u)=0
$$

Comme $u$ est supposé à divergence nulle, cette équation est formellement équivalente à "l'équation de transport" (ou "équation d'advection")

$$
\partial_{t} c+u \cdot \nabla c=0
$$

qui exprime que $u$ "se conserve" le long des trajectoires du champ $u$. La première équation présente toutefois l'avantage de garder un sens si l'on suppose seulement que l'énergie de $u$ est finie. On peut incorporer les données initiales et finales, respectivement notées $c^{0}(x)$ et $c^{T}(x)$, ainsi que la condition d'imperméabilité du bord, dans la formulation faible:

$$
\begin{gathered}
\int_{Q}\left(\partial_{t} f(t, x)+u(t, x) . \nabla f(t, x)\right) c(t, x) d t d x= \\
\int_{D}\left(c^{T}(x) f(T, x)-c^{0}(x) f(0, x)\right) d x
\end{gathered}
$$

pour toute fonction $f(t, x)$ régulière sur $Q$. Elle implique la "conservation de la masse" $\int_{D} c(t, x) d x$ au cours du temps (en prenant $f(t, x)$ indépendant de $x$ ) et, en particulier, la condition de compatibilité sur les données

$$
\int_{D} c^{T}(x) d x=\int_{D} c^{0}(x) d x
$$

\section{Remarques}

Si $u(t, x)$ est Lipschitz continue en $x$, avec une constante de Lipschitz intégrable en temps, $c$ est entièrement déterminé par sa donnée sur $D$ à un instant $t$ fixé quelconque -et en particulier par sa donnée initiale ou sa donnée finale-, en vertu du théorème de Cauchy-Lipschitz. Le même résultat reste vrai sous l'hypothèse sensiblement plus faible que les dérivées $\partial_{i} u(t, x)$ (au sens des distributions) sont intégrables sur $Q$, suivant la théorie (récente) de DiPerna et Lions [10]. Sous ces mêmes hypothèses, la propriété que $c(t, x)$ est binaire est garantie en tous temps dès qu'elle est assurée à l'instant initial (ou final). En revanche, en dessous de ce degré de régularité de $u$ (que nous ne supposerons d'ailleurs pas dans la suite), les propriétés d'unicité et de binarité peuvent être violées. Notons enfin que, suivant la régularité supposée de $u$, des conditions de compatibilité supplémentaires doivent être ajoutées. Ainsi, lorsque $c^{0}$ est la fonction indicatrice d'un sous domaine régulier $D^{0}$ de $D$ on voit que $c^{T}$ doit être l'indicatrice d'un domaine $D^{T}$ difféomorphe à $D^{0}$ si $u$ est supposé de classe $C^{1}$ ou plus, et au moins homéomorphe dans le cas Lipschitzien. En particulier, dans ces cas, la connexité éventuelle de $D^{0}$ doit être aussi vérifiée par $D^{T}$.

\section{Le problème variationnel avec données incomplètes}

Etant données les données compatibles d'un traceur $c$ aux instants initial et final $c^{0}(x)$ et $c^{T}(x)$, on cherche un champ de vitesse $u(t, x)$ de carré intégrable sur $Q$, à divergence nulle 
et les valeurs $c(t, x)$ du traceur aux instants intermédiaires $0<t<T$, de sorte à minimiser l'énergie de $u$, i.e. l'intégrale sur $Q$ de son carré (ou encore le carré de sa norme $L^{2}$ sur $Q$ ), sous la contrainte que la formulation faible (3) est satisfaite. Cela revient à résoudre

$$
\inf _{u, c} \sup _{f} L(u, c, f)
$$

où, le "lagrangien" $L$ est défini par :

$$
L(u, c, f)=\int_{Q}\left(\frac{1}{2}|u|^{2}-\left[\partial_{t} f+u . \nabla f\right] c\right) d t d x-\int_{D}\left(c^{T} f(T, .)-c^{0} f(0, .)\right) d x,
$$

et $u$ décrit l'ensemble des champs de vitesse à divergence nulle, tangents au bord, de carré sommable sur $Q, c$ celui des fonctions binaires sur $Q$ et $f$ celui des fonctions régulières sur $Q$.

L'existence d'une solution optimale n'est en rien évidente. En effet, le Lagrangien ne correspond pas à un problème de minimisation convexe, à cause de la présence du produit cu.

\section{Une nouvelle formulation du problème variationnel}

Un procédé formel permet d'associer naturellement au problème variationnel un problème convexe (dont on peut montrer l'existence d'une solution et établir les équations d'optimalité en toute rigueur, comme on le verra dans la sous-section suivante). L'idée est simple. Notons $c_{1}(t, x)=c(t, x)$ et introduisons le traceur "complémentaire" $c_{2}(t, x)=1-c_{1}(t, x)$ de sorte que

$$
\sum_{\alpha} c_{\alpha}(t, x)=1
$$

où l'indice $\alpha$ vaut 1 ou 2 . Associons à chaque traceur $c_{\alpha}$ le champ de quantité de mouvement

$$
m_{\alpha}(t, x)=c_{\alpha}(t, x) u(t, x) .
$$

On a alors la loi de conservation

$$
\partial_{t} c_{\alpha}+\nabla \cdot m_{\alpha}=0
$$

qui peut être écrite sous forme faible en incorporant valeurs initiales et finales des traceurs

$$
\begin{gathered}
\int_{Q}\left(\partial_{t} f(t, x) c_{\alpha}(t, x)+\nabla f(t, x) \cdot m_{\alpha}(t, x)\right) d t d x= \\
\int_{D}\left(c_{\alpha}^{T}(x) f(T, x)-c_{\alpha}^{0}(x) f(0, x)\right) d x .
\end{gathered}
$$

Notons en premier lieu qu'en sommant les équations (9) et en utilisant (7) on retrouve automatiquement que $u$ est à divergence nulle. On peut donc remplacer cette dernière condition et la loi de conservation du traceur (1) par la paire (9),(7). On a ensuite l'identité suivante:

$$
|u|^{2}=\sum_{\alpha} c_{\alpha}|u|^{2}=\sum_{\alpha} \frac{\left|m_{\alpha}\right|^{2}}{c_{\alpha}} .
$$


Observons que la fonction $k(c, m)=|m|^{2} /(2 c)$ peut être étendue pour tout réel $c$ et vecteur $m \in \mathbb{R}^{d}$ si on pose

$$
k(c, m)=\sup _{a+|b|^{2} / 2 \leq 0} a c+b . m,
$$

où $a$ et $b$ décrivent respectivement $R$ et $\mathbb{R}^{d}$. Elle est convexe, prend ses valeurs dans $[0,+\infty]$ et vaut $+\infty$ si et seulement si ou bien $c<0$ ou bien $c=0$ et $m \neq 0$.

Toutes ces propriétés nous conduisent à introduire un nouveau Lagrangien, qu'on notera encore $L$, dépendant cette fois des variables $c_{\alpha}, m_{\alpha}$, d'un multiplicateur de Lagrange $p(t, x)$ pour prendre en compte la contrainte (7) et de multiplicateurs $\phi_{\alpha}(t, x)$ pour chacune des lois (9), soit:

$$
\begin{aligned}
& L\left((c, m, \phi)_{\alpha}, p\right)=\sum_{\alpha}\left[\int_{Q}\left(k\left(c_{\alpha}, m_{\alpha}\right)-p c_{\alpha}-\partial_{t} \phi_{\alpha} c_{\alpha}-m_{\alpha} . \nabla \phi_{\alpha}\right) d t d x\right. \\
&\left.+\int_{D}\left(c_{\alpha}^{T} \phi_{\alpha}(T, .)-c_{\alpha}^{0} \phi_{\alpha}(0, .)\right) d x\right]+\int_{Q} p d t d x .
\end{aligned}
$$

Le problème posé au départ équivaut au nouveau problème variationnel:

$$
\inf _{(c, m)_{\alpha}} \sup _{\left(\phi_{\alpha}\right)_{, p}} L\left((c, m, \phi)_{\alpha}, p\right)
$$

où l'on impose aux fonctions $c_{\alpha}$ d'être binaires. Notons que l'indice $\alpha$, qui jusqu'à présent prenait les valeurs 1 et 2 , peut tout aussi bien varier de 1 à $N$, pour n'importe quel choix de $N \geq 2$. C'est ce que nous ferons dorénavant. (Cela revient à "suivre" $N-1$ traceurs au lieu d'un seul.) On utilisera parfois le mot "phase" pour parler de chaque traceur indexé par $\alpha$.

\section{Le problème convexifié}

Observons que si nous relâchons la contrainte de binarité, nous obtenons un problème de minimisation convexe que nous appelerons dans la suite problème "convexifié". Dans ce cas, les fonctions $c_{\alpha}$ prennent automatiquement leurs valeurs dans l'intervalle [0,1] puisque les valeurs (strictement) négatives sont "pénalisées" grace à la fonction $k$ et que leur somme par rapport à $\alpha$ vaut 1 . Ainsi, la différence entre le problème originel et le problème convexifié est que l'on autorise dans le second les inconnues $c_{\alpha}$ à prendre comme valeurs toutes les combinaisons convexes des valeurs binaires 0 et 1 . Calculons d'abord formellement les conditions d'optimalité du problème convexifié. En annulant successivement les "dérivées fonctionelles"

$$
\frac{\delta L}{\delta m_{\alpha}}, \frac{\delta L}{\delta c_{\alpha}}, \frac{\delta L}{\delta \phi_{\alpha}}, \frac{\delta L}{\delta p},
$$

on retrouve bien entendu les contraintes avec les deux dernières dérivées et avec les deux premières

$$
\begin{gathered}
m_{\alpha}=c_{\alpha} \nabla \phi_{\alpha}, \\
\partial_{t} \phi_{\alpha}+\frac{1}{2}\left|\nabla \phi_{\alpha}\right|^{2}+p=0 .
\end{gathered}
$$

Notons une différence capitale par rapport au problème originel: lorsque les $c_{\alpha}$ prennent des valeurs non binaires, c'est-à-dire lorsqu'il y a plusieurs phases $\alpha$ présentes au même point $(t, x)$, alors chacune d'elles y possède son propre champ de vitesse

$$
v_{\alpha}=\nabla \phi_{\alpha} .
$$


Les équations décrivent plutôt un écoulement multiphasiques de fluides immiscibles qu'un écoulement unique transportant divers traceurs! Par un calcul encore très formel, on peut réécrire (15) en en prenant le gradient, en multipliant par $c_{\alpha}$ et en utilisant l'équation de conservation (9), pour obtenir une équation de "conservation de la quantité de mouvement" pour chaque phase $\alpha$ :

$$
\partial_{t}\left(c_{\alpha} v_{\alpha}\right)+\nabla \cdot\left(c_{\alpha} v_{\alpha} \otimes v_{\alpha}\right)+c_{\alpha} \nabla p=0 .
$$

\section{Solutions du problème convexifié}

Les équations (14),(15) ont été obtenues formellement et posent d'évidentes difficultés dans les cas extrèmes où les $c_{\alpha}$ prennent les valeurs 0 et 1 , qui sont précisément les valeurs intéressantes! Il faut donc procéder avec soin pour obtenir le résultat rigoureux suivant:

Théorème 1.1 Supposons que $D=(\mathbb{R} / \mathbb{Z})^{d}$ et que les données $c_{\alpha}^{0}(x)$ et $c_{\alpha}^{T}(x)$ prennent leurs valeurs dans $[0,1]$ et vérifient les conditions de compatibilité

$$
\int_{D} c_{\alpha}^{T}(x) d x=\int_{D} c_{\alpha}^{0}(x) d x, \quad \sum_{\alpha} c_{\alpha}^{0}=\sum_{\alpha} c_{\alpha}^{T}=1 .
$$

Alors il existe au moins une solution $(c, m)_{\alpha}$ optimale. Pour chaque $\alpha$, on peut écrire

$$
m_{\alpha}(t, x)=c_{\alpha}(t, x) v_{\alpha}(t, x),
$$

où $v_{\alpha}$ est bien défini dans $L^{2}\left(Q, c_{\alpha}(t, x) d t d x\right)$ (c'est-à-dire de carré sommable par rapport au poids $\left.c_{\alpha}\right)$. Il existe une distribution $p(t, x)$ sur $Q$, unique à l'addition près d'une distribution ne dépendant que de la variable $t$, ne dépendant que des données, telle que, dans l'intérieur de $Q(17)$ est satisfaite au sens des distributions, $\nabla p(t, x)$ étant une mesure localement bornée et le produit $c_{\alpha} \nabla p$ pouvant être défini sans ambiguité. Si de plus l'intérieur de $\{(t, x) \in$ $\left.Q ; \quad c_{\alpha}(t, x)>0\right\}$ est non vide, alors $v_{\alpha} y$ dérive d'un potentiel, i.e. vérifie (16).

Ce théorème améliore sensiblement un résultat antérieur de [5]. C'est un corollaire direct d'un résultat plus général, prenant en compte aussi le cas de données lagrangiennes complètes, que nous évoquerons plus loin et qui est annoncé dans [8] et prouvé dans [9].

\section{Le cas de solutions binaires et les équations d'Euler}

On a vu que, pour le problème convexifié, chaque phase $\alpha$ se voit attribuer son propre champ de vitesse $v_{\alpha}$ (bien défini " $c_{\alpha}(t, x) d t d x$ - presque partout"). S'il se trouve, par chance, que les fonctions $c_{\alpha}$ optimales ne prennent que les valeurs 0 et 1 , elles seront forcément solutions du problème de minimisation originel, puisque le problème convexifié n'est rien d'autre que le problème de minimisation originel où l'on s'est affranchi de la contrainte de binarité. Examinons alors les propriétés qu'on peut tirer du résultat rigoureux de la soussection précédente. Contrairement au cas général, on peut maintenant définir un unique champ de vitesse $u(t, x)$ commun à tous les phases $\alpha$, en posant

$$
u(t, x)=\sum_{\alpha} c_{\alpha}(t, x) v_{\alpha}(t, x)
$$

On a alors bien

$$
m_{\alpha}=c_{\alpha} u
$$

et

$$
\partial_{t} c_{\alpha}+\nabla \cdot\left(u c_{\alpha}\right)=0
$$


ce qui assure que chaque $c_{\alpha}$ est bien un traceur. En sommant en $\alpha$ ces équations ainsi que les équations (17), on obtient

$$
\nabla . u=0, \quad \partial_{t} u+\nabla \cdot(u \otimes u)+\nabla p=0,
$$

qui ne sont rien d'autres que les équations d'un fluide parfait incompressible. Ainsi on a reconstruit un champ de vitesse solution (faible) des équations d'Euler à l'aide de données lagrangiennes incomplètes, ce qui était notre but. En examinant d'un peu plus près les conditions d'optimalité, on voit qu'il s'agit d'un type très particulier de solutions des équations d'Euler. Supposons en effet que les ensembles $Q_{\alpha}=\left\{(t, x) \in Q ; c_{\alpha}(t, x)>0\right\}$ sont (à des ensembles de mesure de Lebesgue nulle près) des ouverts, alors dans chacun de ces ensembles le champ de vitesse est potentiel, autrement dit le tourbillon (i.e. le rotationnel de $u$ ) y est nul. Cela signifie que l'écoulement reconstitué est composé de zones potentielles séparées par des "nappes tourbillonaires" où se concentre le tourbillon.

\section{Quand les solutions sont-elles binaires?}

A priori, les solutions $c_{\alpha}$ du problème convexifié n'ont pas de raison d'être binaires. C'est un problème tout à fait ouvert de savoir quand la binarité a lieu, même dans le cas où les données sont générées par une solution, disons classique, des équations d'Euler! Ainsi la pertinence du problème convexifié par rapport au problème originel peut être mise en doute. En revanche, en suivant en gros la même stratégie, on va parvenir à un résultat beaucoup plus satisfaisant dans le cas de données lagrangiennes complètes.

\subsection{Données lagrangiennes complètes}

Nous supposons à présent qu'au temps final $T$ la position $h(a) \in D$ de la particule fluide issue du point $a$ au temps 0 est connue pour chaque $a \in D$. Bien entendu, comme précédemment, suivant la régularité supposée du champ de vitesse $u$, les conditions de compatibilité sur la donnée $h$ sont plus ou moins fortes. La condition la plus faible, due à la conservation des volumes impliquées par l'incompressibilité supposée du fluide, est:

$$
\int_{D} f(h(a)) d a=\int_{D} f(a) d a
$$

pour toute fonction continue $f$ sur $D$. Cela signifie que $h$ (à une modification éventuelle sur une partie Lebesgue négligeable de $D$ près) appartient à l'ensemble $S(D)$ des applications boréliennes de $D$ dans lui-même vérifiant (21), c'est-à-dire "conservant la mesure de Lebesgue". C'est ensemble est un semi-groupe pour la loi de composition, dont l'identité est l'élément neutre. Ce n'est pas un groupe car (21) n'implique pas que $h$ soit inversible, même presque partout (comme le montre l'exemple élémentaire $h(x)=2 x(\bmod 1)$ pour $D=[0,1])$. C'est en revanche une partie fermée de l'espace ambiant $L^{2}\left(D, \mathbb{R}^{d}\right)$, inclus dans une sphère, comme on peut le vérifier aisément. Si on suppose le champ suffisamment régulier, de classe $C^{1}$ par exemple, $h$ doit appartenir au sous-ensemble de $S(D)$ constitué des difféomorphismes de $D$ ayant un déterminant jacobien identiquement égal à 1, que nous noterons $G(D)$ (bien que souvent noté $S \operatorname{Dif} f(D))$. Cet ensemble est un groupe pour la loi de composition. On peut montrer que, muni de la norme $L^{2}\left(D, \mathbb{R}^{d}\right), S(D)$ est le complété de $G(D)$ (voir [13] dans le cas de $\left.D=[0,1]^{d}\right)$ dès que $d>1$.

A l'instar du cas de données lagrangiennes incomplètes, on peut chercher un champ de vitesse $u$ d'énergie minimale compatible avec $h$. La compatibilité avec $h$ est difficile à exprimer en fonction de $u$, car elle revient à imposer que, pour chaque $a \in D$ la solution $g(t, a)=x(t)$ de l'équation différentielle $d x / d t=u(t, x)$ telle que $g(0, a)=a$ doit satisfaire $g(T, a)=h(a)$. C'est là une contrainte très indirecte imposée au champ $u$. Il est bien plus commode de tout 
exprimer en termes "lagrangiens", en considérant comme inconnue le "flot" $(t, a) \rightarrow g(t, a)$. La correspondance entre $u$ et $g$ est biunivoque dès que $u$ est assez régulier et $t \rightarrow g(t,$.$) peut$ être vue comme une trajectoire sur le groupe $G(D)$. On a de plus l'identité

$$
\int_{Q}|u(t, x)|^{2} d t d x=\int_{Q}\left|\partial_{t} g(t, a)\right|^{2} d t d a
$$

(en notant que $\partial_{t} g(t, a)=u(t, g(t, a))$ et que $g(t,$.$) conserve la mesure de Lebesgue).$

\section{Reformulation du problème}

Une technique de convexification a été proposée par l'auteur dans [1] puis développée dans [4]. Elle repose sur le concept de "flot généralisé", une variante des "mesures de Young" bien connues en homogénéisation [16] [15]. (En fait, ce concept avait été introduit par Shelukhin peu avant pour les écoulements compressibles [14].) Néanmoins, en vue d'obtenir des conditions d'optimalité précises, la meilleure technique de convexification est, à nos yeux, de suivre l'exemple des données lagrangiennes incomplètes, en passant en quelque sorte du discret (un nombre fini de "traceurs") au continu (un continuum de "traceurs" infinitésimaux). Chaque particule $a$ transportée par le flot $g(t, a)$ peut en effet être vue comme une sorte de traceur valant 0 en tout point distinct $x \neq g(t, a)$ et $+\infty$ en $x=g(t, a)$. En termes mathématiques, on associe à chaque particule $a$ la masse de Dirac

$$
c(t, x, a)=\delta(x-g(t, a)) .
$$

Elle est solution (au sens faible des distributions) de l'équation de continuité (1) des traceurs, rencontrée dans la première section. On associe aussi à chaque particule un champ de quantité de mouvement qui est la mesure (vectorielle)

$$
m(t, x, a)=u(t, x) c(t, x, a)=\partial_{t} g(t, a) \delta(x-g(t, a)) .
$$

On peut alors réécrire, d'un coup pour toutes les particules à la fois, l'équation de continuité

$$
\begin{gathered}
\int_{Q^{\prime}}\left(\partial_{t} f(t, x, a) d c(t, x, a)+\nabla_{x} f(t, x, a) \cdot d m(t, x, a)\right) \\
=\int_{D}(f(T, h(a), a)-f(0, a, a)) d a
\end{gathered}
$$

où on demande à la fonction test $f(t, x, a)$ d'avoir ses dérivées partielles en $(t, x)$ continues sur $Q^{\prime}=Q \times D$. On a utilisé la notation $\nabla_{x}$ pour bien prendre en compte que dorénavant les fonctions test dépendent non seulement des variables usuelles $(t, x)$ mais aussi des particules étiquetées par leur position initiale $a$. On a aussi utilisé les notations $d c$ et $d m$ pour les intégrales relatives aux mesures $c$ (positive) et $m$ (vectorielle). L'incompressibilité se traduit par la condition

$$
\int_{Q^{\prime}} f(t, x) d c(t, x, a)=\int_{Q} f(t, x) d t d x
$$

pour toute fonction continue $f$ sur $Q$, ou encore, avec une notation plus compacte $\int_{D} c(t, x, d a)$ $=1$. Enfin l'énergie du fluide est, à l'instar du cas de données incomplètes, donnée par

$$
K(c, m)=\sup _{(A, B)} \int_{Q^{\prime}}(A(t, x, a) d c(t, x, a)+B(t, x, a) \cdot d m(t, x, a))
$$

où $A$ et $B$ décrivent l'ensemble des fonctions continues de $Q^{\prime}$ dans respectivement $R$ et $\mathbb{R}^{d}$ qui vérifient en tous points l'inégalité

$$
A(t, x, a)+\frac{1}{2}|B(t, x, a)|^{2} \leq 0 .
$$


Ainsi on peut reformuler le problème des données complètes comme la recherche de

$$
\inf _{(c, m)} \sup _{(\phi, p)} L(c, m, \phi, p),
$$

où le Lagrangien est à présent donné par

$$
\begin{gathered}
L(c, m, \phi, p)=K(c, m)-\int_{Q^{\prime}}\left[\partial_{t} \phi(t, x, a)+p(t, x)\right] d c(t, x, a) \\
-\int_{Q^{\prime}} \nabla_{x} \phi(t, x, a) \cdot d m(t, x, a)+\int_{D}(\phi(T, h(a), a)-\phi(0, a, a)) d a+\int_{Q} p(t, x) d t d x
\end{gathered}
$$

avec la contrainte que la mesure $c(t, x, a)$ est, pour chaque $(t, a)$, une masse de Dirac en $x$.

\section{Le problème convexifié}

Comme dans la première section, il ne nous reste plus qu'à relâcher la contrainte pour $c(t, x, a)$ d'être une masse de Dirac en $x$. Observons que la seule finitude de $K(c, m)$ suffit à faire de $c$ une mesure positive (au sens large) et de $m$ une mesure vectorielle absolument continue par rapport à $c$, qu'on peut écrire

$$
m(t, x, a)=v(t, x, a) c(t, x, a) .
$$

La densité vectorielle $v$ appartient à l'ensemble des champs de carré sommable sur $Q^{\prime}$ par rapport à la mesure $c$ et l'énergie $K(c, m)$ se réécrit alors simplement comme

$$
K(c, m)=\int_{Q^{\prime}} \frac{1}{2}|v(t, x, a)|^{2} d c(t, x, a) .
$$

Le problème convexifié consiste donc précisément à résoudre (29) pour le Lagrangien (30), en recherchant $c$ et $m$ comme mesures sur $Q^{\prime}$, respectivement à valeurs réelles et vectorielles, c'est-à-dire (par le théorème d'identification de Riesz) comme des formes linéaires continues sur l'espace des fonctions continues sur le compact $Q^{\prime}$ à valeurs respectivement réelles et vectorielles. On montre alors (dans le cas du tore $\mathbb{R}^{d} / \mathbb{Z}^{d}$ ), l'équivalent du théorème 1.1. (Voir [8] pour un énoncé détaillé.) Les équations d'optimalité généralisent les équations d'Euler et sont en fait les équations régissant un plasma quasi-neutre discutées dans [7] et étudiées dans les travaux de Grenier [11], [12].

Le problème convexifié est lié aux solutions régulières des équations d'Euler par le résultat de cohérence suivant (cf. [9]) :

Théorème 1.2 Supposons D convexe. Soit $(u, p)$ une solution classique de l'équation d'Euler des fluides parfaits incompressibles et soit g le flot associé. Posons $h(a)=g(T, a)$. Soit $\lambda$ le sup en $(t, x)$ sur $Q$ de la plus grande valeur propre de la matrice hessienne de pression $D^{2} p(t, x)$. Si

$$
\lambda T^{2}<\pi^{2},
$$

alors la paire $(c, m)$ associée à u par (23), (24) est l'unique solution du problème convexifié avec données $T$ et $h$.

\section{Remarques}

Ainsi, en résolvant le problème convexifié on retrouve automatiquement les solutions locales classiques des équations d'Euler. Il est amusant de noter que l'inégalité sur le temps $T$ est saturée par l'exemple élémentaire suivant. Prenons $d=2$ et pour $D$ le disque unité. Considérons le champ de rotation uniforme autour de l'origine $u\left(x_{1}, x_{2}\right)=\left(-x_{2}, x_{1}\right)$, qui est une solution très banale des équations d'Euler, "stationnaire" (i.e. indépendante du temps), dont le champ de pression est $p\left(x_{1}, x_{2}\right)=\frac{1}{2}\left(x_{1}^{2}+x_{2}^{2}\right)$. On s'aperçoit que le temps maximal de strict minimalité est bien donné par $T=\pi$ qui correspond à une demi-rotation du disque. 


\section{Méthodes numériques}

Cette partie décrit brièvement quelques méthodes, décrites en détail dans la thèse de Michel Roesch [13], pour le calcul numérique du problème de données lagrangiennes complètes. (Nous ne discutons pas le cas, pourtant plus important en pratique, de données incomplètes.) Dans le cadre classique on recherche un flot $g$ incompressible, c'est-à-dire une courbe $t \in[0, T] \rightarrow g(t,$.$) sur le groupe G(D)$ des difféomorphismes de $D$ à déterminant jacobien partout égal à 1 , joignant l'identité à la donnée $h$ et de longueur minimale relativement à la métrique héritée de l'espace de Hilbert ambiant $L^{2}\left(D, \mathbb{R}^{d}\right)$. On se tourne donc naturellement vers des techniques de discrétisation lagrangienne. Soulignons que, du point de vue de la résolution numérique du problème de valeurs initiales pour les équations d'Euler, la pratique est très différente et on choisit en général de discrétiser l'espace des champs de vitesses (c'est-à-dire en termes géométriques, "l'algèbre de Lie" plutôt que le "groupe de Lie"). On peut élargir la discrétisation lagrangienne au sous-groupe $S(D)$ des applications boréliennes conservant $D$ et la mesure de Lebesgue, qui constitue le complété de $G(D)$ pour la norme $L^{2}\left(D, \mathbb{R}^{d}\right)$ dès que $d \geq 2$.

\subsection{Semi-discrétisation en temps}

\section{Le problème semi-discret}

Fixons $N>2$, normalisons le temps final $T=1$ et posons $\Delta t=\frac{1}{N}$. Un flot semi-discret (ou discret en temps) sur l'espace de configuration $G=G(D)$ est défini comme une suite $g^{1}, g^{2}, \ldots, g^{N} \in G$, où $g^{k} \in G$ est la configuration au temps $t_{k}=\frac{k}{N}$. Le problème semidiscrétisé (ou discrétisé en temps) de données lagrangiennes complètes consiste à trouver un flot semi-discret tel que $g^{1}=i d$ et $g^{N}=h$, minimisant

$$
E_{N}\left(g^{1}, g^{2}, \ldots, g^{N}\right)=\frac{N}{2} \sum_{k=2}^{N}\left\|g^{k}-g^{k-1}\right\|_{L^{2}}^{2} .
$$

\section{Projection sur $S(D)$ et décomposition polaire des applications}

Intéressons nous au cas particulier $N=3$, qui nous servira à la résolution du problème semi-discret pour $N>3$ quelconque. On recherche un triplet de configurations $\left(g^{1}, g^{2}, g^{3}\right)$ tel que $g^{1}=i d, g^{3}=h$ minimisant

$$
\left\|g^{3}-g^{2}\right\|_{L^{2}}^{2}+\left\|g^{2}-g^{1}\right\|_{L^{2}}^{2} .
$$

Cela revient à minimiser l'expression $\left\|\frac{1}{2}(h+i d)-g^{2}\right\|_{L^{2}}^{2}$ pour $g^{2} \in G$. La configuration $g^{2}$ obtenue n'est rien d'autre que la projection sur $G$ au sens de la norme de $L^{2}(D)$, de l'application $\frac{1}{2}(h+i d)$. Mais l'existence d'une telle projection n'a rien d'évident, ni du point de vue de l'existence et ni de celui de l'unicité, car $G$ n'est même pas fermé dans $L^{2}\left(D, \mathbb{R}^{d}\right)$ (sauf dans le cas trivial $d=1$ ). La projection $L^{2}$ sur le complété $S(D)$ ne s'annonce guère mieux, car il n'est ni convexe ni compact (puisque contenu dans une sphère). On peut pourtant la définir sans équivoque pour toute application $w \in L^{2}\left(D, \mathbb{R}^{d}\right)$ satisfaisant la condition de nondégénerescence que, pour tout borélien $\omega \subset \mathbb{R}^{d}$ de mesure de Lebesgue nulle, $w^{-1}(\omega)$ est de mesure nulle dans $D$. On a montré en effet [2] [6] qu'il existe alors une unique décomposition de l'application $w$ (appelée décomposition polaire par généralisation du cas bien connu des matrices carrées) sous la forme

$$
w=\nabla \Phi \circ h
$$

où $h$ est un élément de $S(D)$, caractérisée comme unique projection $L^{2}$ de $w$, et $\Phi$ (définie à une constante additive près), est une fonction convexe sur l'enveloppe convexe de $D$ telle que $\nabla \Phi \in L^{2}\left(D, \mathbb{R}^{d}\right)$. 


\section{L'algorithme itératif de semi-discrétisation en temps}

On propose ici un algorithme récursif qui à partir des configurations à deux instants donnés permet de calculer la configuration à l'instant intermédiaire. On suppose que $N$ est de la forme $N=2^{n}+1$, on modifie un peu les notations de sorte que $g^{0}, g^{\frac{1}{N}}, \ldots, g^{\frac{k}{N}}, \ldots, g^{1}$ soient les configurations aux instants respectifs $0, \frac{1}{N}, \ldots, \frac{k}{N}, \ldots, 1$ et on fixe $g^{0}=i d$ et $g^{1}=h$. Dans le cas $N=3$, l'algorithme proposé est, comme on l'a vu, de prendre pour $g^{1 / 2}$ la projection $L^{2}$ sur $S=S(D)$ du point milieu $\left(g^{1}+g^{0}\right) / 2$. On notera $g^{1 / 2}=\operatorname{mil}\left(g^{0}, g^{1}\right)$. Dans le cas général $N>3$, on procède en raffinant successivement la discrétisation temporelle, comme suit. On part de

$$
g^{1 / 2}=\operatorname{mil}\left(g^{0}, g^{1}\right),
$$

puis on passe au niveau suivant en effectuant

$$
g^{1 / 4}=\operatorname{mil}\left(g^{0}, g^{1 / 2}\right), \quad g^{3 / 4}=\operatorname{mil}\left(g^{1 / 2}, g^{1}\right)
$$

et on réactualise

$$
g^{1 / 2}=\operatorname{mil}\left(g^{1 / 4}, g^{3 / 4}\right) .
$$

On peut réitérer ce processus jusqu’à stationnarité (au moins approximative). Ensuite,

$$
\begin{gathered}
g^{1 / 8}=\operatorname{mil}\left(g^{0}, g^{1 / 4}\right), \quad g^{3 / 8}=\operatorname{mil}\left(g^{1 / 4}, g^{1 / 2}\right), \\
g^{5 / 8}=\operatorname{mil}\left(g^{1 / 2}, g^{3 / 4}\right), \quad g^{7 / 8}=\operatorname{mil}\left(g^{3 / 4}, g^{1}\right), \\
g^{1 / 4}=\operatorname{mil}\left(g^{1 / 8}, g^{3 / 8}\right) \quad g^{1 / 2}=\operatorname{mil}\left(g^{3 / 8}, g^{5 / 8}\right), \\
g^{3 / 4}=\operatorname{mil}\left(g^{5 / 8}, g^{7 / 8}\right),
\end{gathered}
$$

et caetera.

\subsection{Discrétisation combinatoire}

Il a été observé dans [3] qu'un espace de discrétisation naturel pour $S(D)$ (qui est le complété de $G(D)$ pour la norme $L^{2}$ dès que $d \geq 2$ ), est le groupe $P(D)$ de toutes les "permutations" de $D$, construit comme suit, dans le cas particulier $D=[0,1]^{d}$ qu'on choisit pour fixer les idées. Pour chaque entier fixé $p \geq 0$, On discrétise le domaine spatial $D=[0,1]^{d}$ en $2^{p d}$ cubes $D_{i}$ de centres respectifs $x_{i}, 1 \leq i \leq 2^{p d}$ de taille $1 / 2^{p}$. Si $\sigma$ est une permutation de $\left\{1, \ldots ., 2^{p d}\right\}$, on lui associe de façon unique l'élément $g \in S(D)$ défini presque partout par

$$
\forall x \in D_{i}, g_{\sigma}(x)=x-x_{i}+x_{\sigma(i)} .
$$

On obtient ainsi, pour chaque $p$, une partie $P_{p}(D)$ de $S(D)$, qui est un groupe pour la loi de composition, et on note $P(D)$ leur réunion pour tous les $p$. On obtient une discrétisation complète du problème de données complètes, en substituant $P_{p}(D)$, pour un $p$ fixé, à $S(D)$ (ou $G(D)$ ). Le problème élémentaire de projection sur $P_{p}(D)$ se réduit au problème combinatoire suivant, dit d'affectation, qui consiste, pour un vecteur $w \in \mathbb{R}^{2^{\text {pd }}}$ donné, à trouver une permutation $\sigma$ minimisant le "coût d'affectation"

$$
\sum_{i=1}^{2^{p d}} c_{i, \sigma(i)}
$$

où la "matrice de coût" $c_{i j}$ est donnée par

$$
c_{i j}=\left|w_{i}-x_{j}\right|^{2}
$$


Remarquons le cas particulier où $d=1$, qui n'est pas dénué d'intérêt malgré les apparences. En effet une donnée finale $H$ en dimension un, i.e. un élément de $S([0,1])$, peut être étendue trivialement en une donnée en dimension trois $h \in S\left([0,1]^{3}\right)$ en posant simplement $h\left(x_{1}, x_{2}, x_{3}\right)=\left(H\left(x_{1}\right), x_{2}, x_{3}\right)$. Il s'agit d'une donnée généralisée. Ainsi l'intérêt d'étudier numériquement des exemples de données unidimensionnelles est réel. (On en trouvera la justification théorique dans [8], [9].) Or, l'algorithme de projection discret est particulièrement simple en dimension 1, car il se ramène à un tri, comme on peut s'en rendre compte aisément à partir de l'expression de $c_{i j}$. Le coût de calcul est alors presque optimal, de l'ordre de $O(N \log (N))$ avec $N=2^{p}$. (Il n'en est à notre connaissance pas de même en dimension supérieure ou le coût passe à $O\left(N^{2} \log (N)\right)$ avec $N=2^{\text {pd }}$.) Pour plus de détails, on peut se référer à [3]. Quelques exemples de résultats numériques sont fournis dans [13].

\subsection{Une deuxième méthode de discrétisation en espace}

Le coût de l'algorithme combinatoire étant élevé pour $d>1$, voici une méthode alternative de discrétisation complète, introduite dans [13]. On ne décrit que la séquence élémentaire du schéma semi-discrétisé en temps. A partir de deux configurations classiques $g^{0}$ et $g^{1}$ de $G(D)$, correspondant aux instants $t_{0}$ et $t_{1}=t_{0}+\Delta t$, on cherche à déterminer une configuration $g^{1 / 2}$ intermédiaire en $t_{1 / 2}=t_{0}+\Delta t / 2$. On utilise la propriété de groupe de $G(D)$ pour se ramener au cas où $g^{0}$ est l'identité. Faisons un développement de Taylor en temps autour de $t=0$ de la solution recherchée $t \rightarrow g(t, a)$ entre $t=0$ et $t=\Delta t$ (supposé petit). On obtient

$$
g(t, a)=g(0, a)+t u(0, a)-\frac{t}{2} \nabla p(0, a)+O\left(t^{3}\right)
$$

en supposant que $g$ est bien solution des équations d'Euler (écrite en coordonnées lagrangiennes)

$$
\partial_{t t} g(t, a)=-\nabla p(t, g(t, a))
$$

avec $g(0, a)=a$ et où $\partial_{t} g(0, a)=u(0, a)$ est le champ de vitesse au temps $t=0$. (Ceci est cohérent avec le théorème 1.2.) On doit donc avoir

$$
g^{1}(a)=a+\Delta t u(0, a)-\frac{\Delta t^{2}}{2} \nabla p(0, a)+O\left(\Delta t^{3}\right) .
$$

et, au temps intermédiaire $t=\Delta t / 2$,

$$
g^{1 / 2}(a)=a+\frac{\Delta t}{2} u(0, a)-\frac{\Delta t^{2}}{8} \nabla p(0, a)+O\left(\Delta t^{3}\right) .
$$

Pour éliminer la pression, on utilise que $\nabla \cdot u(0, a)=0$. On en tire

$$
-\frac{\Delta t^{2}}{2} \nabla p(0, a)=g^{1}(a)-a+\Delta t u(0, a)+O\left(\Delta t^{3}\right)
$$

d'où

$$
-\frac{\Delta t^{2}}{2} \Delta p(0, a)=\nabla \cdot\left(g^{1}(a)-a\right)+O\left(\Delta t^{3}\right) .
$$

On posera donc,

$$
g^{1 / 2}(a)=\frac{1}{2}\left(a+g^{1}(a)\right)-\frac{1}{4} \nabla(-\Delta)^{-1} \nabla \cdot\left(g^{1}(a)-a\right),
$$

ce qui nous fournit un schéma (formellement) consistant avec les équations d'Euler. Observons qu'un calcul direct montre que l' application $g^{1 / 2}$ conserve la mesure de Lebesgue sur $D$ à $O\left(\Delta t^{3}\right)$ près. La résolution pratique est effectuée dans [13] pour le cas bidimensionnel du tore $D=\mathbb{R}^{2} / \mathbb{Z}^{2}$, maillé de manière uniforme. Voici la cellule de base du calcul

1. On se ramène à l'identité en composant (approximativement) par $\left(g^{0}\right)^{-1}$. 
2. On calcule

$$
g^{1 / 2}(a)=\frac{1}{2}\left(a+g^{1}(a)\right)-\frac{1}{4} \nabla(-\Delta)^{-1} \nabla\left(g^{1}(a)-a\right),
$$

à l'aide d'une transformée de Fourier discrète (FFT).

3 . On recompose (approximativement) le résultat par $g^{0}$.

Notons que le maillage n'autorise que des compositions approximatives à l'aide d'interpolations (par opposition au schéma combinatoire précédemment décrit). On renvoie à [13] pour les problèmes que cela induit et pour une illustration numérique, où Roesch reconstitue (avec succès) l'écoulement de Beltrami, solution des équations d'Euler sur $\mathbb{R}^{2} / \mathbb{Z}^{2}$, donnée par la "fonction de courant"

$$
\psi\left(x_{1}, x_{2}\right)=\frac{1}{\pi} \sin \left(\pi x_{1}\right) \sin \left(\pi x_{2}\right)
$$

et le champ de pression

$$
p=\frac{1}{4}\left(\sin ^{2}\left(\pi x_{1}\right)+\sin ^{2}\left(\pi x_{2}\right)\right),
$$

sur tout intervalle de temps $[0, T]$ assez petit pour que la condition (33) du théorème 1.2 soit satisfaite.

\section{Références}

[1] Y. BRENIER, J. of the AMS 2, (1990), 225-255.

[2] Y. Brenier, Comm. Pure Appl. Math. 64, (1991), 375-417.

[3] Y. Brenier, Comp. Meth. in Appl. Mech. 75, (1989), 325-332.

[4] Y. Brenier, The Dual Least Action Problem for an Ideal, Incompressible fluid, Arch. Rational Mech. Anal. 122, no. 4, (1993), 323-351.

[5] Y. Brenier, A homogenized model for vortex sheets, (to appear in Arch. Rational Mech.) (1997), Rapport LMENS 95-10 (May 95).

[6] Y. Brenier, On the motion of an ideal incompressible fluid, Partial differential equations of elliptic type, (Cortona, 1992), 123-148, Sympos. Math., XXXV, Cambridge Univ. Press, Cambridge, 1994.

[7] Y. Brenier, Quelques lois de conservations issues de modèles cinétiques, Journées Équations aux Dérivées Partielles, (Saint-Jean-de-Monts, 1995), Exp. No. I, École Polytechnique, Palaiseau, 1995.

[8] Y. Brenier, Homogénéisation variationnelle des équations d'Euler, Séminaire EDP 96-97, exposé no X, Ecole Polytechnique, 1997.

[9] Y. Brenier, Variational analysis of the Euler incompressible fluid equations, (en préparation).

[10] R. DiPerna \& P.-L. Lions, Invent. Math. 98, (1989), 511-547.

[11] E. Grenier, Oscillations in quasineutral plasmas, Comm. Partial Differential Equations 21, (1996), 363-394.

[12] E. Grenier, Pseudodifferential energy estimates of singular perturbations, (to appear in Comm. Pure and Applied Maths), Rapport LMENS-96-16 (June 96).

[13] M. Roesch, Thèse, Université Paris 6, 1995.

[14] V. Shelukhin, Existence Theorem in the variational problem for compressible inviscid fluids, Manuscripta mathematica 61, (1988), 495-509.

[15] L. TARTAR, The compensated compactness method applied to systems of conservation laws, Systems of nonlinear PDE, NATO ASI series, 1983.

[16] L.C. Young, Lectures on the calculus of variations, Chelsea, New York, 1980. 